\title{
CALIBRATION AND VALIDATION OF A SHARED SPACE MODEL: A CASE STUDY
}

\author{
Bani Anvari \\ Research a Centre for Transport Studies \\ Department of Civil and Environmental Engineering \\ Imperial College London \\ SW7 2BU, UK \\ b.anvari09@imperial.ac.uk
}

\author{
Michael GH Bell \\ Professor of Ports and Maritime Logistics \\ Institute of Transport and Logistics Studies \\ Business School \\ The University of Sydney \\ NSW 2006 Australia \\ michael.bell@sydney.edu.au

\section{Panagiotis Angeloudis} \\ Lecturer in Urban Engineering \\ Centre for Transport Studies \\ Department of Civil and Environmental Engineering \\ Imperial College London \\ SW7 2BU, UK \\ p.angeloudis@imperial.ac.uk
}

\section{Washington Y Ochieng}

Professor in Positioning and Navigation Systems

Centre for Transport Studies

Department of Civil and Environmental Engineering

Imperial College London

SW7 2BU, UK

w.ochieng@imperial.ac.uk

Word count: (Tables: $2 * 250$ + Figures: $7 * 250+$ Abstract: $241+$ Text: $3888+$ References: $853=$ Total: $7232<7,500$ )

Paper submitted for presentation and publication at the 95th Annual Meeting of the Transportation Research Board January11-15, 2016, Washington D.C.

November 20, 2015 


\begin{abstract}
Shared space is an innovative streetscape design which seeks minimum separation between vehicle traffic and pedestrians. Urban design is moving towards space sharing as a means of increasing the community texture of street surroundings. Its unique features aim to balance priorities and allow cars and pedestrians to co-exist harmoniously without the need to dictate behaviour. There is, however, a need for a simulation tool to model future shared space schemes and to help judge if they might represent suitable alternatives to traditional street layouts. This paper builds on the authors' previously published work where a shared space model was presented based on the Social Force Model (SFM). The calibration of this microscopic model was evaluated using data from a shared space link typology of New Road in Brighton (United Kingdom). Here, we collected and analysed data recorded from the shared space scheme of Exhibition Road, London (United Kingdom) which has the same typology. However, there is a higher flow and speed of cars and more segregation between pedestrians and cars on Exhibition Road. Data from pedestrian and vehicle movements and their interaction has been collected and analysed. The rule-based SFM for shared space modelling is calibrated and validated using the real data. Based on the results, it can be concluded that shared space schemes are context-dependent and factors such as the infrastructural design of the environment and the flow and speed of pedestrians and vehicles affect the willingness to share space.
\end{abstract}

Keywords: Shared space, Microscopic model, Social Force Model, Calibration, Validation 


\section{INTRODUCTION}

Shared space is an innovative approach to enhance the design of streets and places. This scheme is based on the idea that both pedestrians and vehicles share a single surface with integrated layouts and features encouraging a considerate style of driving to balance priority for all road users (1). The key features of the shared space concept are to reduce clutter on streets and increase the awareness of safety, reduce the separation between vehicle traffic and pedestrians to introduce a degree of uncertainty as to the right-of-way. Further, attractive features are added to the environment for pedestrians to provide pleasurable areas and stimulate pedestrians to walk and cycle to their destinations (2, 3). As a result, shared space designs improve the social context of streets by reducing traffic speed and giving a feeling of comfort for non-motorised users. Vehicle emissions are reduced by decreasing stop-and-go behaviours, property values are increased due to the enhancement of accessibility for pedestrians, and the reduction of clutter increases awareness of safety.

Modelling future shared space schemes helps urban designers as well as public and local authorities to judge whether this concept might represent a suitable alternative to traditional street layouts. Mathematically formulating the behaviour of and interaction between the travel modes/agents - non-motorised (pedestrians) and motorised transport (4-wheeled vehicles) - in order to simulate a shared space scheme is a challenge and requires real world observations. Hence, this paper focuses on the evaluation of a microscopic model for simulating shared space schemes. The implemented model is evaluated by empirical data from a shared space environment in London, United Kingdom.

In Section 2, the latest mathematical models for simulating mixed traffic are thoroughly reviewed. The Social Force Model (SFM) provides a unified theory for explaining both vehicle and pedestrian movements, separately and in interaction with each other; since they have parameters that can be easily interpreted and can describe the largest set of traffic and crowd dynamics. This microscopic model is the core of the model presented in Section 3 with three layers: the trajectory planning, the force based and the rule based layer. Section 4 introduces the shared space scheme of Exhibition Road in London. Data from pedestrians and cars is analysed and used for calibration and validation of our shared space model (see Section 5). The results are discussed and the findings of the paper concluded in Section 6 .

\section{BACKGROUND}

Research on mixed pedestrian and driver traffic has largely focused on empirical studies instead of simulation models (4, 5, 6, 7). There are only a limited number of studies modelling the integration of vehicular traffic and pedestrians. Among these studies, researchers have investigated interactions of pedestrians and drivers at the crossing points of streets: $\mathrm{Li}(\mathbf{8})$ proposed a statistical model for analysing field data on the street-crossing behaviour of pedestrians. He extracted different waiting time distributions for pedestrians before crossing the street. Among pedestrian and vehicle interaction models, Helbing et al. (9) analysed and formulated the interaction of pedestrians with vehicles at crossing sections with a force directed model. Pedestrian arrival rates and safety factors of pedestrian gap acceptance are the main factors in this proposed car-following model. Pretto et al. (10) used a combination of force directed and rule-based approaches for modelling interactions of pedestrians and vehicles at crossing points. Sun et al. (11) defined a gap acceptance model for pedestrian-vehicle interactions on a crosswalk. They proposed a deterministic gap acceptance 
model for pedestrians and a probabilistic gap acceptance model for drivers. A decision making process is modelled using a binary logit model. Sun et al. (12) developed a Pedestrian Crossing Behaviour Virtual Reality System (PCBVRS), based on the traffic microscopic simulation software, VISSIM, to study issues of pedestrian crossing behaviours. Wang et al. (13), meanwhile, used jaywalk data of pedestrians outside crossing facilities and developed a pedestrian gap acceptance model based on a discrete choice approach. Zhang and Chang (14) investigated the use of the CA model for simulating vehicle-pedestrian interactions. A conflict (competition) is detected when a cell is assigned as the target for multiple agents. In this case, the waiting time of an individual is considered as a factor on winning the competition. Ottomanelli et al. (15) proposed a gap acceptance model for interaction of pedestrians and vehicles at crossings. They used the CA model at crossing areas and derived interaction parameters from a probabilistic distribution. CA models make simulation of mixed traffic computationally efficient, since a rule set is applied over many time steps rather than finding solutions for differential equations. They are discrete in nature, however, and it may be difficult to explain the cause of an unexpected macroscopic behaviour when it emerges from locally defined interaction. Pedestrian traffic is analysed in (16, 17) using VISSIM, where vehicle and pedestrian modes operate independently and are controlled by the traffic signals at potential conflicting areas with vehicle priority.

In this paper, we build on our previously published work (18) where we presented a shared space model based on the Social Force Model (SFM) and calibrated and validated the microscopic model based on data from New Road in Brighton (united Kingdom). Here, we collected and analysed data recorded from the shared space scheme of Exhibition Road in London (United Kingdom). Both shared space schemes are of the same typology. However, as Exhibition Road is located in South Kensington, one of the most cultural destinations in London attracting over nine million visitors a year (19), there is a higher flow and speed of cars and more segregation between pedestrians and cars. Data from pedestrian and vehicle movements and their interaction has been collected and analysed. Our rule-based SFM for shared space modelling (18, 20, 21, 22) is calibrated and validated using the real data. We believe that the contribution of this paper will make it possible to evaluate the performance of new designs with different traffic volumes and simulate a shared space system with suitable and essential characteristics of pedestrians and car drivers.

\section{OVERVIEW OF THE SHARED SPACE MODEL}

In this section, an overview of the mathematical shared space model is given. The architecture of the model is composed of three layers: the trajectory planning, the force based and the rule based layer. The Social Force Model (SFM) builds the fundamental basis of the framework. More details can be found in (18).

\subsection{Modelling vehicular agents}

Similar to pedestrians $\alpha$, a car $\gamma$ is introduced by an ellipse with the radius $r_{\gamma}\left(\varphi_{\gamma U}\right)$. The radius $r_{\gamma}\left(\varphi_{\gamma U}\right)$ depends on the angle $\varphi$ between the desired direction of a car $\gamma$ and the direction of a close-by pedestrian $U=\alpha$ or car $U=\delta$. The radius of the ellipse $r_{\gamma}\left(\varphi_{\gamma U}\right)$ in polar coordinates is 
described by Equation 1 .

$$
r_{\gamma}\left(\varphi_{\gamma U}\right)=\frac{w}{\sqrt{1-\varepsilon^{2} \cos ^{2}\left(\varphi_{\gamma U}\right)}}, \text { where } \varepsilon=\frac{\sqrt{l^{2}-w^{2}}}{l},
$$

where $w$ is the width and $l$ the length of a car.

\subsection{Trajectory planning by distance map}

In order to define intermediate destinations for each agent in the SFM to avoid obstacles in the environment, a global shortest path strategy is implemented based on a priori knowledge. In this process, the floor area is divided into cells and the distance values for all the obstacle cells are assigned a large number and the empty cells are set to zero. Then, a distance map is generated through iterations. The distance values of direct eight neighbourhood cells are added starting from the destination point to the starting point. This is achieved by calculating the Variant 2 flood fill $\left(D^{V 2}\right)$ based on a combination of Manhattan metric $D^{\mathrm{M}}$ and Chessboard metric $D^{\mathrm{C}}(23)$ as in Equation 2.

$$
D^{V 2}=(\sqrt{2}-1) D^{m}+D^{C}, \text { where }\left\{\begin{array}{l}
D^{\mathrm{M}}=\sum_{i}\left|\delta x_{i}\right|+\sum_{i}\left|\delta y_{i}\right| \\
D^{\mathrm{C}}=\sum_{i} \max \left(\left|\delta x_{i}\right|,\left|\delta y_{i}\right|\right) \\
D^{\mathrm{m}}=D^{\mathrm{M}}-D^{\mathrm{C}}
\end{array}\right.
$$

The model's prior geometrical information about the environment and the shortest path to the destination is given before running the simulation. Intermediate destinations are automatically generated for the agents, according to distance map calculations and collision checks with the obstacles. The agent navigates via these intermediate destinations.

\subsection{Force based modelling}

As mentioned earlier, the core of this microscopic model is based on the SFM developed by Helbing et al. (24). The original SFM was initialised by Lewin's (25) idea of social science that behavioural changes are driven by social forces. Helbing explained this idea mathematically and applied the concept to pedestrians' dynamics. Since cars and pedestrians move within shared space environments with equivalent priority, the SFM for pedestrians is considered here and applied to a model for cars and pedestrians.

The sum of the force terms exerted to a car $\gamma$ from a pedestrian $\alpha$, a boundary $b$ and another vehicle $\delta$ can be seen in Equation 3 .

$$
\frac{d \vec{v}_{\gamma}(t)}{d t}=\vec{f}_{\gamma}^{0}+\sum_{\delta(\delta \neq \gamma)} \vec{f}_{\gamma \delta}+\sum_{\alpha} \vec{f}_{\gamma \alpha}^{s o c}+\sum_{b} \vec{f}_{\gamma b}+\vec{\xi}
$$

Equivalent to Equation 3, the sum of the forces exerted to a pedestrian $\alpha$ from a car $\gamma$, a boundary $b$ and another pedestrian $\beta$ can be seen in Equation 4.

$$
\frac{d \vec{v}_{\alpha}(t)}{d t}=\vec{f}_{\alpha}^{0}+\sum_{\alpha(\alpha \neq \beta)} \vec{f}_{\alpha \beta}+\sum_{\alpha} \vec{f}_{\alpha \gamma}+\sum_{b} \vec{f}_{\alpha b}+\vec{\xi}
$$


The first terms $\left(\vec{f}_{\gamma}^{0}, \vec{f}_{\alpha}^{0}\right)$ are driving forces which encourages the agent (pedestrian or car) to move towards its destination with a desired speed that is adapted to the actual velocity within their reaction time. The interaction between road users is captured by adding three different types of forces (socio-psychological, following and physical forces) in the second and third terms of Equations 3 and 4

$$
\begin{gathered}
\vec{f}_{\gamma \delta}=\vec{f}_{\gamma \delta}^{s o c}+\vec{f}_{\gamma \delta}^{\text {following }} \\
\vec{f}_{\alpha \beta}=\vec{f}_{\alpha \beta}^{s o c}+\vec{f}_{\alpha \beta}^{\text {physical }} \\
\vec{f}_{\alpha \gamma}=\vec{f}_{\alpha \gamma}^{s o c}+\vec{f}_{\alpha \gamma}^{\text {physical }}
\end{gathered}
$$

One type of force is the socio-psychological force, $\vec{f}^{s o c}$, which is a repulsive force to keep a certain distance from nearby users. This force is defined by an exponential function to the power of distance from other users (before having any physical contact with each other):

$$
\begin{gathered}
\vec{f}_{\gamma \delta}^{s o c}=A_{\gamma \delta} e^{\frac{r_{\gamma \delta}-d \gamma \delta}{B_{\gamma \delta}}} \vec{n}_{\gamma \delta} F_{\gamma \delta} \\
\vec{f}_{\gamma \alpha}^{s o c}=A_{\gamma \alpha} e^{\frac{r \gamma \alpha-d \gamma \alpha}{B \gamma \alpha}} \vec{n}_{\gamma \alpha} F_{\gamma \alpha} \\
\vec{f}_{\alpha \beta}^{s o c}=A_{\alpha \beta} e^{\frac{r_{\alpha \beta}-d \alpha \beta}{B \alpha \beta}} \vec{n}_{\alpha \beta} F_{\alpha \beta} \\
\vec{f}_{\alpha \gamma}^{s o c}=A_{\alpha \gamma} e^{\frac{r \alpha \gamma-d \alpha \gamma}{B \alpha \gamma}} \vec{n}_{\alpha \gamma} F_{\alpha \gamma}
\end{gathered}
$$

$\vec{n}$ is the normalised vector pointing from another road user (car or pedestrian) to an agent. An effective field of view is included in the form factor $F$ for road users interactions. $A$ and $B$ are are parameters that represent the interaction strength and interaction range of the repulsive force $\vec{f}^{s o c}$ which require calibration. The interaction range $A$ describes the amplitude of a force in the centre mass and it affects how quickly the exerted force decreases with distance. The interaction strength parameter $A$ represents the amount of influence that a force has and is dependent from $B$.

The physical interaction force acts on pedestrian $\alpha$ only in case of physical contact such as panic situations. In general, pedestrians try to avoid physical injuries by pushing other pedestrians in shared space environments.

Another type of force is called the following force $\vec{f}_{\gamma \delta}^{\text {following }}$ which is to capture drivers queuing behaviour in assumed lanes. The empirical data gathered from observations of shared space schemes shows that cars tend to merge into assumed lanes created by car drivers.

The forth term of Equations 3 and $4\left(\vec{f}_{\gamma b}, \vec{f}_{\alpha b}\right)$ is a perpendicular force to the surface of an obstacle to address the obstacle repulsive effects on road users. A random fluctuation force $\vec{\xi}$ is also added to the sum of the exerted forces in Equations 3 and 4 to present velocity fluctuation due to diverse behaviours of road users. Hence, the fluctuation force resolves these deadlocks due to entirely oppositional velocities with minimal influence on the sum of the forces exerted to an agent. More details about the formulation of these forces can be found in (18).

\subsection{Rule based constraints}

Constraining the flexibility of car motions is addressed in this model by a relation between the steering angle and speed considering the centrifugal acceleration expressed by the driver. Hav- 
ing described the key characteristics of shared space by the SFM in the previous sections, there are some potential road conflicts that might occur by following the SFM exclusively which are predicted based on based on agents states and resolved with a combination of speed change and correction of heading direction. By using a conflict avoidance strategy, left-hand traffic is also introduced for car-car interactions when passing in opposite directions (22).

\section{CASE STUDY: EXHIBITION ROAD, LONDON}

\subsection{Geographical integration and video monitoring of the shared space scheme}

Data from the shared space link typology of Exhibition Road (London) is used to calibrate and validate the mathematical model summarised in Section 3. Exhibition Road is located in South Kensington, one of the most cultural destinations in London. This road is located between many famous museums and institutions which attract over nine million visitors a year (19).

The streetscape is one of the busiest roads in South Kensington for both pedestrians and vehicles. The shared space scheme includes a single surface with street de-cluttering, access restrictions, provision of parking locations, loading zones, and bus-stop facilities and was completed in December 2011. The behaviour of pedestrians and vehicles was monitored by CCTV cameras and a camcorder from different angles. A bottom-up approach was followed for the data collection process in order to reduce operating costs with willing to repeat this procedure. Videos were recorded with CCTV cameras $(960 \times 536$ pixels) and a digital camera (Panasonic HDC-HS60, $1920 \times 1080$ pixels) at $30 \frac{\mathrm{frames}}{\mathrm{s}}$. On Exhibition Road, the pedestrian density on the carriageway is lower than on the footway for most of the day. The traffic flow is up to 547 motorized vehicles and 3388 pedestrians during peak hours.

The behaviour of pedestrians and cars was monitored with two CCTV cameras on Thursday, 15 December 2011 during the peak hour from 01:00pm to 02:00pm was chosen (see Figure 1 (a)-(d)). The digital camera was mounted and fixed at an elevation of about $12 \mathrm{~m}$ for the same peak hour on Thursday, 16 February 2012 as shown in Figure 11(e) and (f).

\subsection{Analysis of extracted data}

From the recorded video data during the peak hour, speed and acceleration distributions and trajectories were extracted using the Trajectory Extractor software (26) and superimposed onto the real map. This data is defined as the performance indicators to be reproduced by the shared space model. Data of agents were extracted at a time step of $1 \mathrm{~s}$. The new mathematical model calculates forces every $0.1 \mathrm{~s}$. Hence, the extracted trajectories were resampled to $0.1 \mathrm{~s}$ intervals which correspond to a frame rate of $10 \frac{\text { frames }}{\mathrm{s}}$ using a linear interpolation. The tracked trajectories of pedestrians and cars on Exhibition Road for the digital camera are illustrated in Figure 2, Table 1 summarises the findings of the speed and acceleration distributions of road users on Exhibition Road.

\section{CALIBRATION AND VALIDATION}

As described in Section 3, there are a number of parameters within the formulation of the shared space model for pedestrians and cars. Some parameters depend on the perception, psychological 


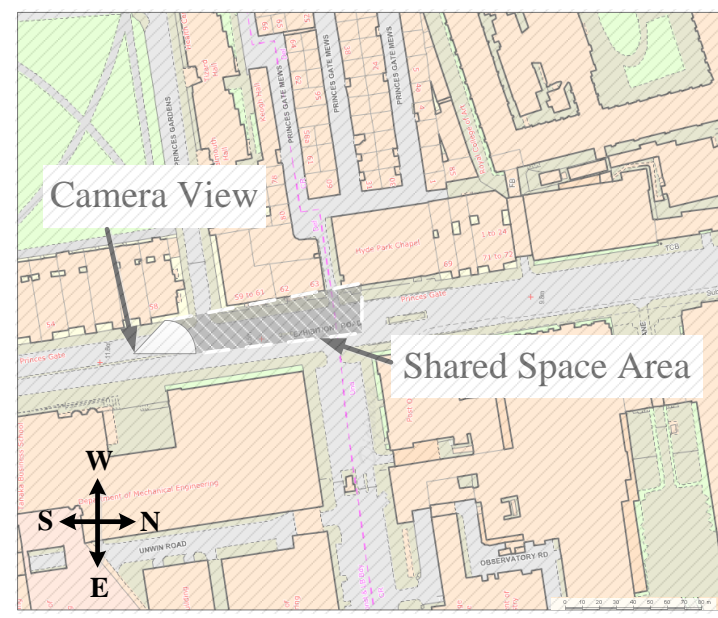

(a)

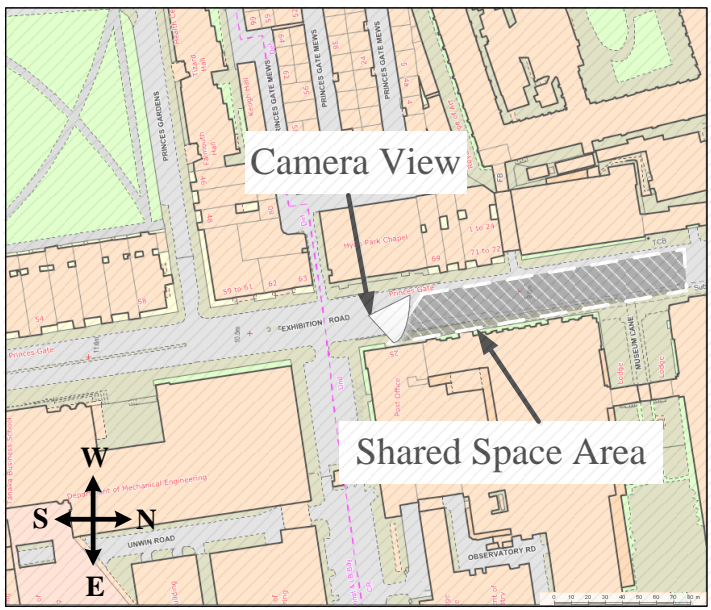

(c)

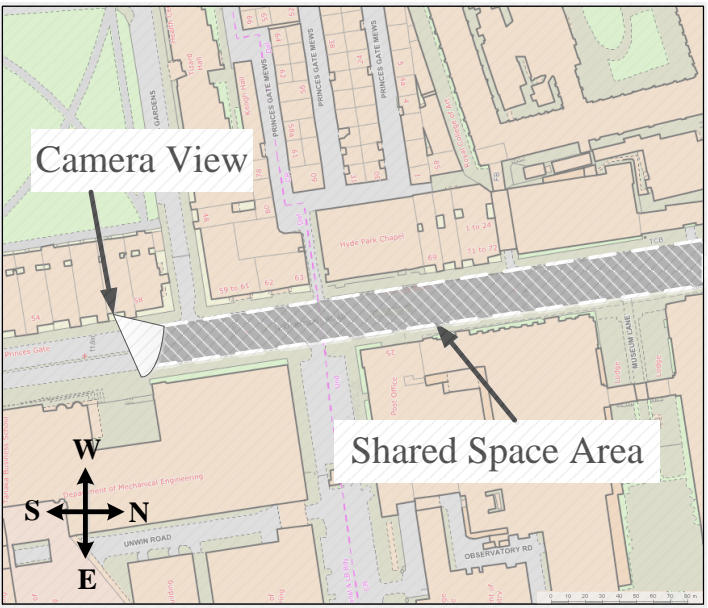

(e)

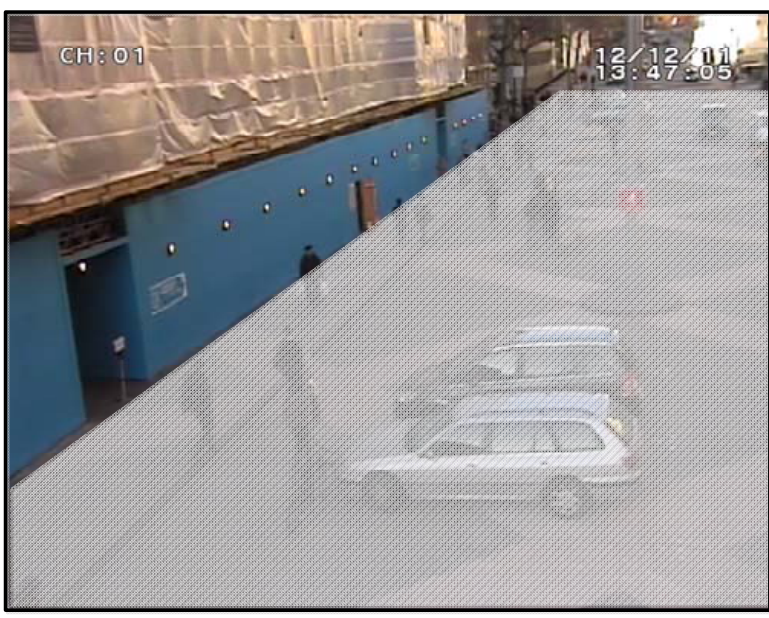

(b)

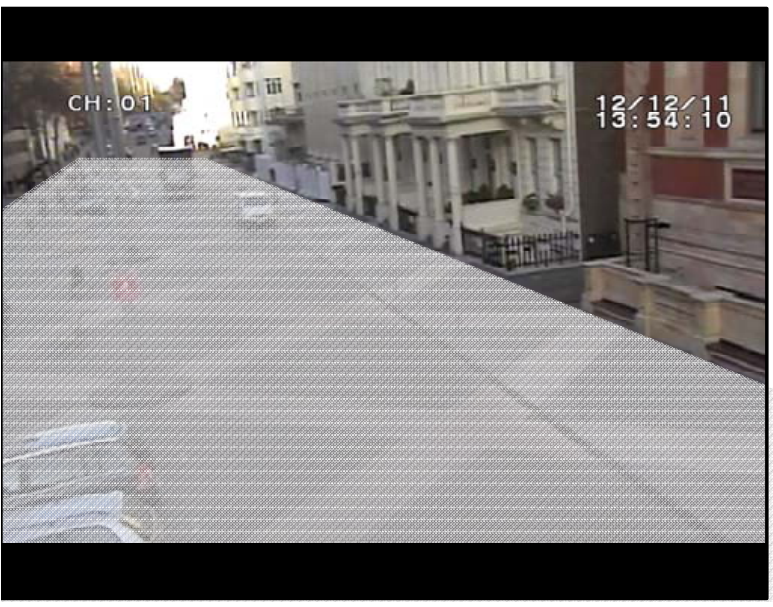

(d)

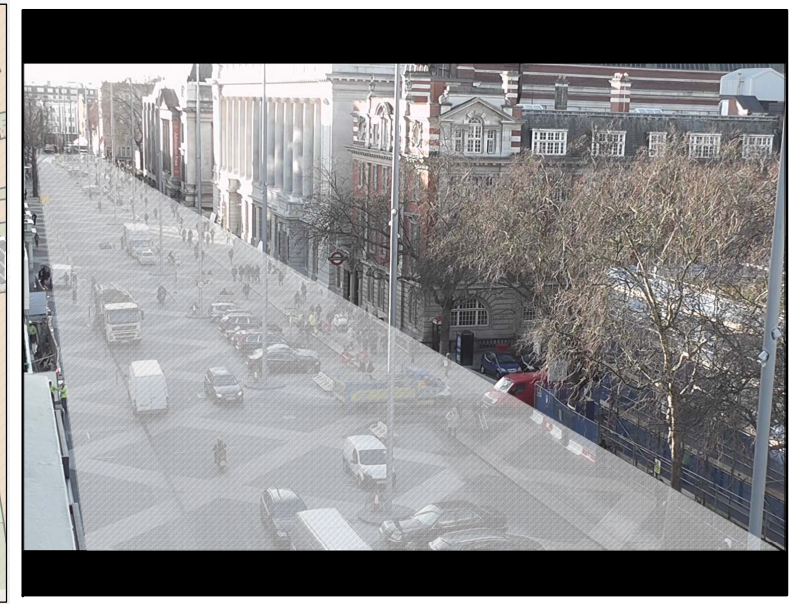

(f)

FIGURE 1 : Shared space of Exhibition Road (London, UK) (a) Top view of area and camera angle, (b) Camera view of CCTV camera \#1, (c) Top view of area and camera angle, (d) Camera view of CCTV camera \#2, (e) Top view of area and camera angle and (f) Camera View of Digital camera 


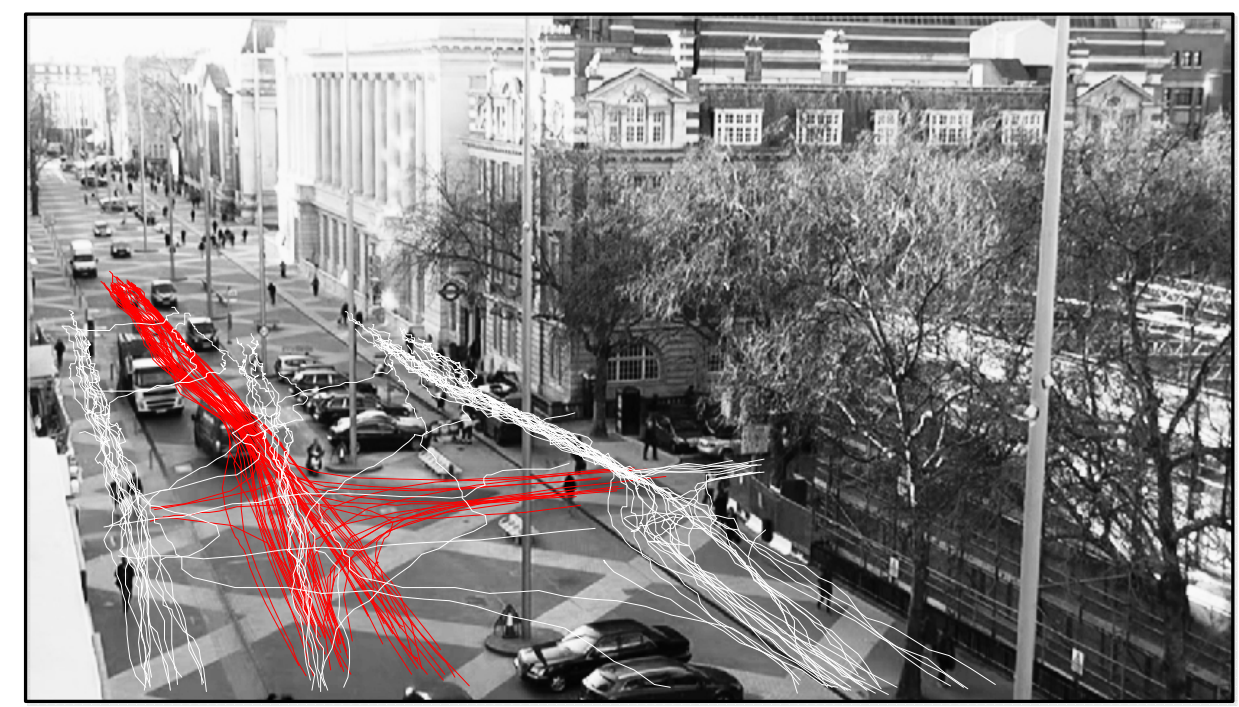

FIGURE 2 : Trackings of 70 pedestrians and 70 cars with the digital camera on Exhibition Road (London, UK)

motivations and social behaviours of road users and they were assigned to a value according to previous studies $(27,28)$. The relaxation time of pedestrians $\tau_{\alpha}=0.3 \mathrm{~s}$ and cars $\tau_{\gamma}=2.4 \mathrm{~s}$, the anisotropy form factor $\lambda=0.2$, the obstruction effect constants $k=1 \frac{\mathrm{kg}}{\mathrm{s}^{2}}$ and $\kappa=1.8 \frac{\mathrm{kg}}{\mathrm{ms}}$, the effective view angle $\vartheta=10^{\circ}$, the safe time headway $T_{\gamma}=0.7 \mathrm{~s}$, the braking time $\tau_{\gamma}^{\prime}=0.77 \mathrm{~s}$ and the minimal vehicle distance $d_{\gamma \delta}^{m}=1.38 \mathrm{~m}$. The simulation included cars of an average size, $w=1.8 \mathrm{~m}$ and $l=4.8 \mathrm{~m}$, and pedestrians with the average shoulder width of $0.5 \mathrm{~m}$ according to (24). The desired speed of pedestrians was set to $1.3 \frac{\mathrm{m}}{\mathrm{s}}$ according to the maximum observed speed for pedestrians and $8.9 \frac{\mathrm{m}}{\mathrm{s}}$ was assigned for the desired speed of cars based on the maximum speed limit in shared space schemes. Maximum acceleration and deceleration limits were also assigned to road users based on the observed data on Exhibition Road.

The interaction ranges $B$ and interaction strengths $A$ for pedestrians and cars have been calibrated using the minimising deviation method (18, 29).

TABLE 1 : Speed and acceleration distributions of the three data sets for each traffic mode on Exhibition Road (London, UK)

\begin{tabular}{|c|c|c|c|c|}
\hline & \multicolumn{2}{|c|}{ Mean speed in $\left[\frac{\mathrm{m}}{\mathrm{s}}\right]\left(\sigma\right.$ in $\left.\left[\frac{\mathrm{m}}{\mathrm{s}}\right]\right)$} & \multicolumn{2}{|c|}{ Mean acceleration in $\left[\frac{\mathrm{m}}{\mathrm{s}^{2}}\right]\left(\sigma\right.$ in $\left.\left[\frac{\mathrm{m}}{\mathrm{s}^{2}}\right]\right)$} \\
\hline & Pedestrians & Cars & Pedestrians & Cars \\
\hline CCTV camera \#1 & $1.42(0.68)$ & - & $-0.01(0.67)$ & - \\
\hline CCTV camera \#2 & - & $6.67(2.76)$ & - & $-0.06(1.38)$ \\
\hline Digital camera & $1.22(0.63)$ & $8.21(3.15)$ & $-0.00013(0.55)$ & $-0.07(1.85)$ \\
\hline
\end{tabular}




\subsection{Hybrid Calibration Methodology}

The minimising deviation approach aims at minimising the deviations between real and simulated pedestrian and car trajectories. This method measures the relative distance of simulated results to real data according to different parameter sets and captures the magnitude of goodness of fit. The likelihood function shows how matching the simulation results are with respect to observed data. A distribution is produced by minimising the square distance between real and simulated data. The relative distance error is defined in Equation 7)(29).

$$
E=\frac{\left\|r_{U}^{\text {simulated }}(t+T)-r_{U}^{\text {tracked }}(t+T)\right\|}{\left\|r_{U}^{\text {tracked }}(t+T)-r_{U}^{\text {tracked }}(t)\right\|}
$$

where $r_{U}$ is the position of an agent $U, t$ is the starting time of the simulation and $T$ is the duration of the simulation. Averaging the relative distance errors over all simulations allows calculating the fitness level of that particular parameter set. A hybrid method using empirical and simulated trajectories is used for the calibration of parameters. Once the video tracking is completed, certain scenarios which include interactions between agents are chosen. For each pedestrian or car, a virtual pedestrian or car is assigned in the simulation domain. A simulation is initiated according to real data in which one agent (pedestrian or car) is moving with the new mathematical model while the others are moving according to the extracted trajectories. This procedure is repeated for every agent $U$ at different starting times $t^{0}$ in a chosen scenario. After each run, the relative distance error is calculated based on Equation 7. The procedural steps for each simulation can be summarised as follows:

1. Defining the infrastructural environment based on the video.

2. Assigning a desired speed, a starting point and an end point for one virtual agent according to the extracted trajectory.

3. Defining the trajectories and speeds of the surrounding agents based on the tracked trajectory.

4. Running the simulation where the virtual agent is moving and interacting with other agents based on the force directed model over $T$.

5. Determining the average relative distance error between the simulated and tracked trajectories.

After running the simulation over different starting times for different agents, the relative distance error in determined and the average $E$ for all users over different starting times is taken as the "fitness" of the parameter set.

\subsection{Calibration and Validation Results}

Interaction strength $A$ and interaction range $B$ have been calibrated by the empirical data of the shared space scheme of Exhibition Road. Figure 3 presents the resulting fitness values as a function of different combinations of interaction strength $A$ and interaction range $B$. Different sets of 

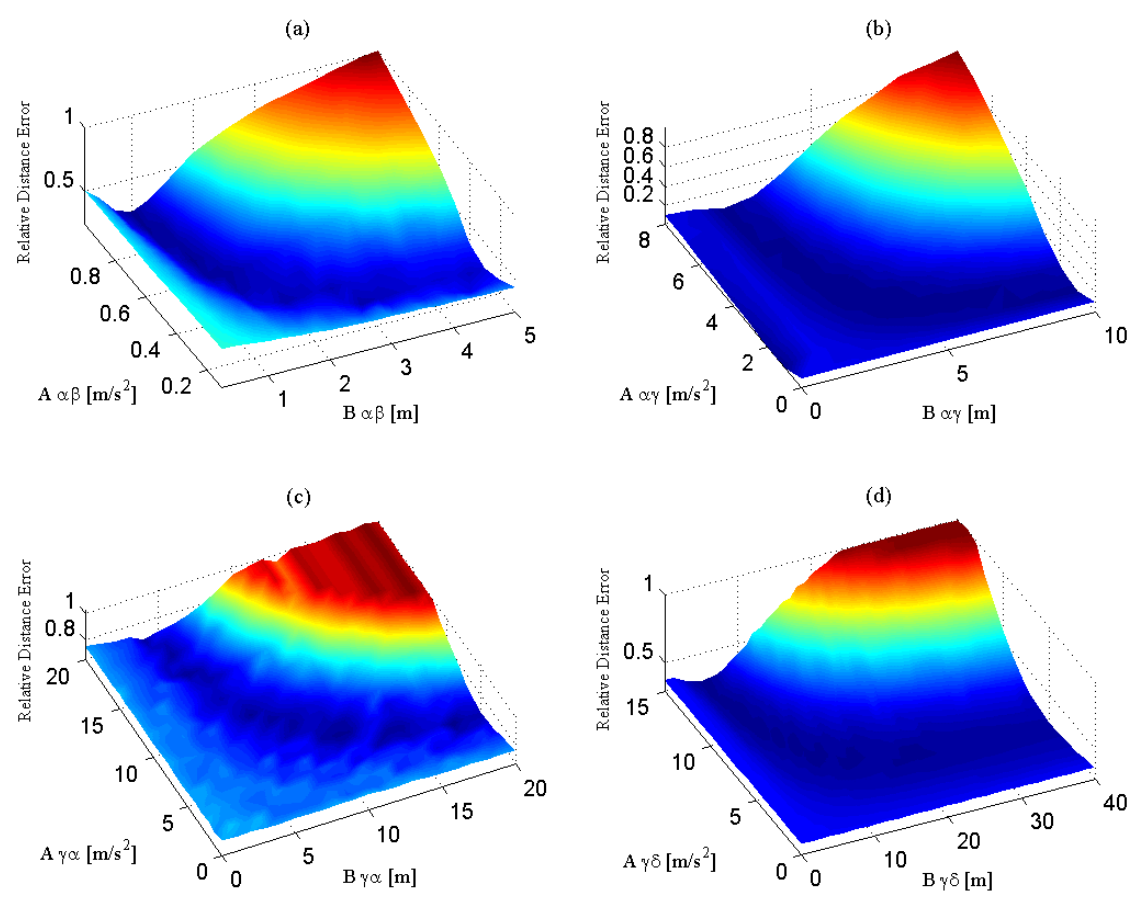

FIGURE 3 : Fitness surface for parameter $A$ and $B$ for (a) Pedestrian-pedestrian-interactions, (b) Pedestrian-car-interactions (c) Car-pedestrian-interactions (d) Car-car-interactions of Exhibition Road

scenarios were used for pedestrian-pedestrian, pedestrian-car, car-pedestrian and car-car interactions. The local minima show the best fitness for the corresponding choice of $A$ and $B$. The travel time and total distance travelled by each agent is compared between the simulated and empirical data in order to find the best combination. Table 2 shows the resulting parameters for Exhibition Road.

For the quantitative assessment (30, 31), the performance indicators obtained by the empirical data of road user behaviours during mixed traffic conditions were compared with the outcomes of the model. The common approach of quantitative validation is to show that the simulation model is able to reproduce real world data after calibration. The shared space model was validated by comparing speed and acceleration distributions and trajectories of real world data to the simu-

TABLE 2 : A summary of the parameters from the calibration process of Exhibition Road (London, UK)

\begin{tabular}{llll}
\hline Interacting road users & $\mathrm{A}\left[\frac{\mathrm{m}}{\mathrm{s}^{2}}\right]$ & $\mathrm{B}[\mathrm{m}]$ & Fitness \\
\hline \hline Pedestrian-pedestrian & {$[0.8 \pm 0.1]$} & {$[1 \pm 0.25]$} & 0.47 \\
Pedestrian-car & {$[3 \pm 1]$} & {$[4 \pm 1]$} & 0.49 \\
Car-pedestrian & {$[7 \pm 1]$} & {$[11 \pm 1]$} & 0.59 \\
Car-car & {$[8 \pm 1]$} & {$[12 \pm 1]$} & 0.40 \\
\hline
\end{tabular}




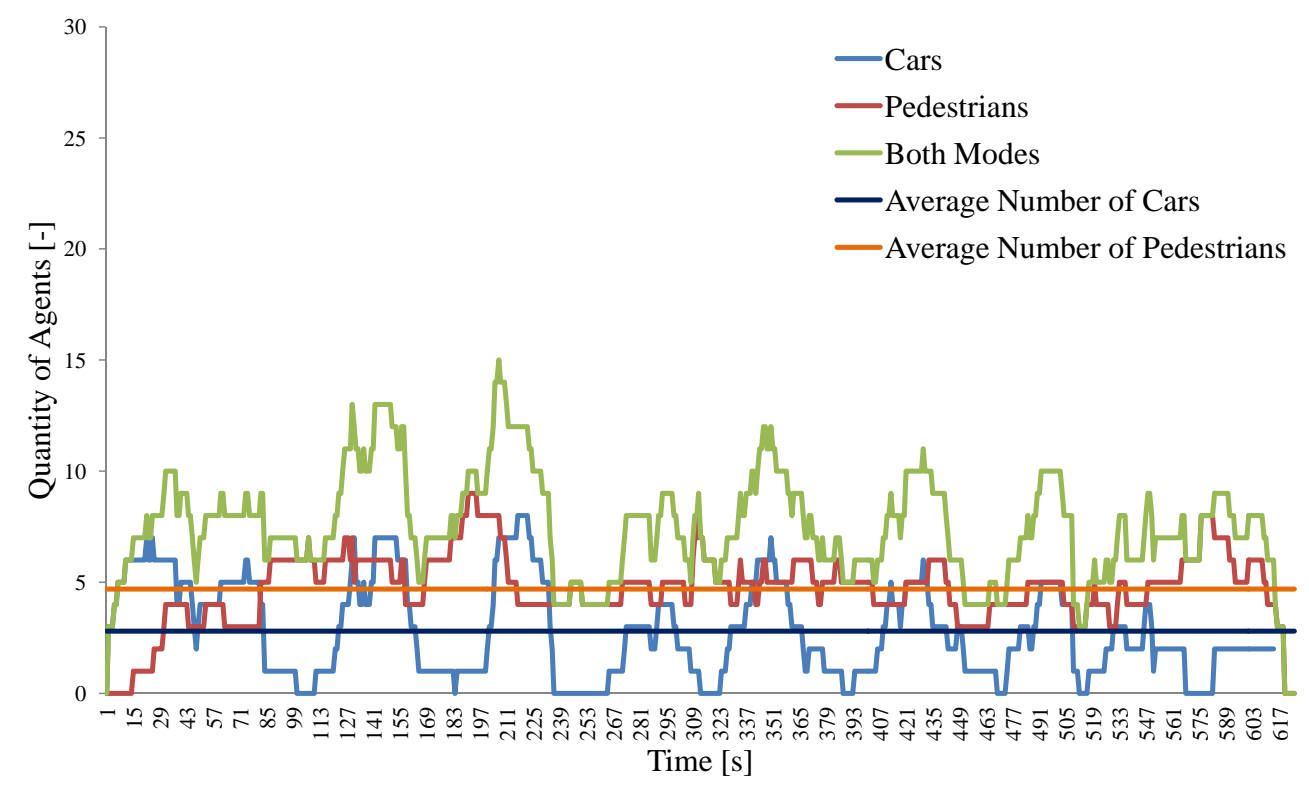

FIGURE 4 : Traffic demand of tracked road users on Exhibition Road (London, UK)

lation results on Exhibition Road. A simulation environment is defined according to the recorded layout of Exhibition Road. Pedestrians and cars are free to move across this shared surface. Traffic demand from the observed data is shown in Figure 4. In Figure 5, the real and simulated trajectories of agents within a time period are plotted. A comparison of speed and acceleration analysis of the real data and simulation results for cars and pedestrians is shown in Figure 6 and 7 .

\section{DISCUSSION AND CONCLUSIONS}

The mixed traffic, microscopic shared space model based on the SFM (18) was calibrated and validated using data from the shared space scheme of Exhibition Road in London. This environment is a link typology which is identical to the one of New Road (Brighton, UK). A detailed evaluation of the latter scheme can be found in (18).

In this paper, we presented an overview of the mathematical shared space model. Using data from CCTV cameras and a digital camera, data from pedestrians and cars was recorded and analysed. Based on the empirical data, the key characteristics of Exhibition Road and differences to New Road (Brighton) can be concluded as follows:

- The density of pedestrians is much higher on the footway than on the carriageway on Exhibition Road.

- The flow of cars on Exhibition Road is up to 10 times more while the pedestrian flow is similar.

- On Exhibition Road, pedestrians mainly remain on the sides of the road whereas cars follow the traditional traffic regulations and stay within assumed lanes.

- The mean speed of pedestrians is similar in both shared space schemes; the mean speed of cars on Exhibition Road is up to 3 times higher than on New Road. 


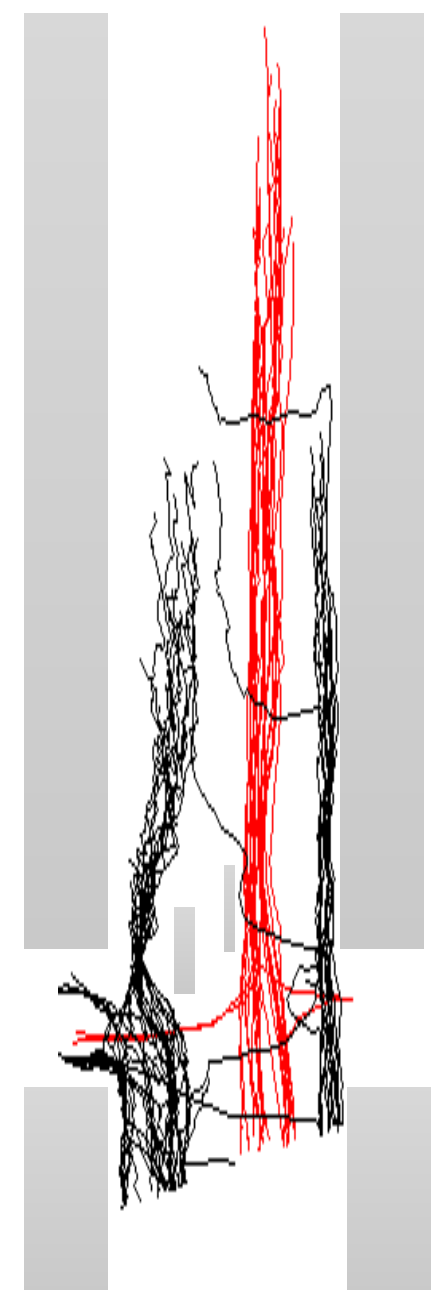

(a)

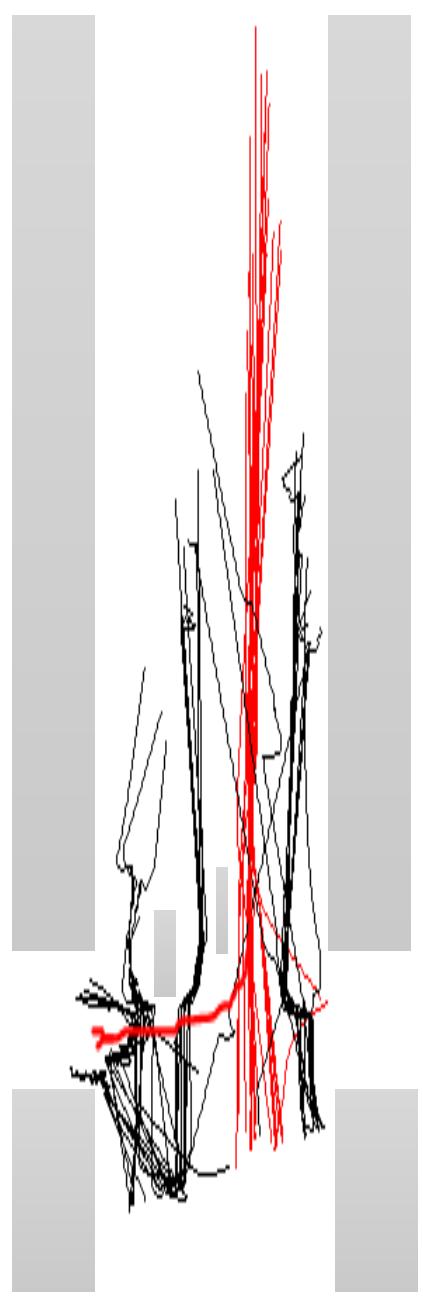

(b)

FIGURE 5 : Pedestrian (black) and car (red) trajectories on Exhibition Road (London, UK) from (a) real data and (b) simulation

Looking at the calibration results of Exhibition Road, combinations of interaction strength $A$ and interaction range $B$ with the best fitness value cover a wider range compared to the results on New Road. This can be explained with the environmental design of New Road which contributes to more interactions at lower speeds compared to Exhibition Road. Comparing the values of the interaction range $B$ between cars and pedestrians, the calibration in this paper results in larger values for agents on Exhibition Road which can be explained by the shared space design - which is more segregated than the one in Brighton. Our calibration results agree with the investigation of Kaparias et al. (32) exploring reaction distances of cars and pedestrians in case of potential collisions on Exhibition Road.

The calibrated shared space model was validated reproducing speed and acceleration distributions of pedestrians and cars obtained by empirical data. The trajectory analysis reproduces the observed tendency of pedestrians and cars to prefer segregation. Agents that are modelled by the new mathematical model strictly follow the shortest path via intermediate destinations. Regarding vehicles, the car-following feature and rule-based constraints have been simulated and agree with 
(a)

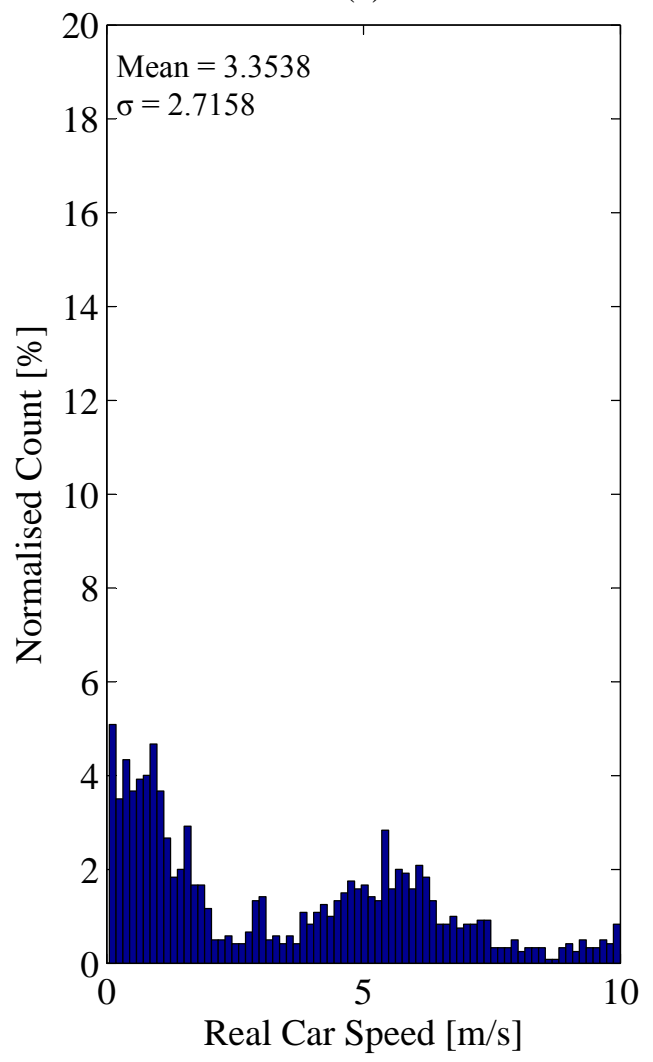

(c)

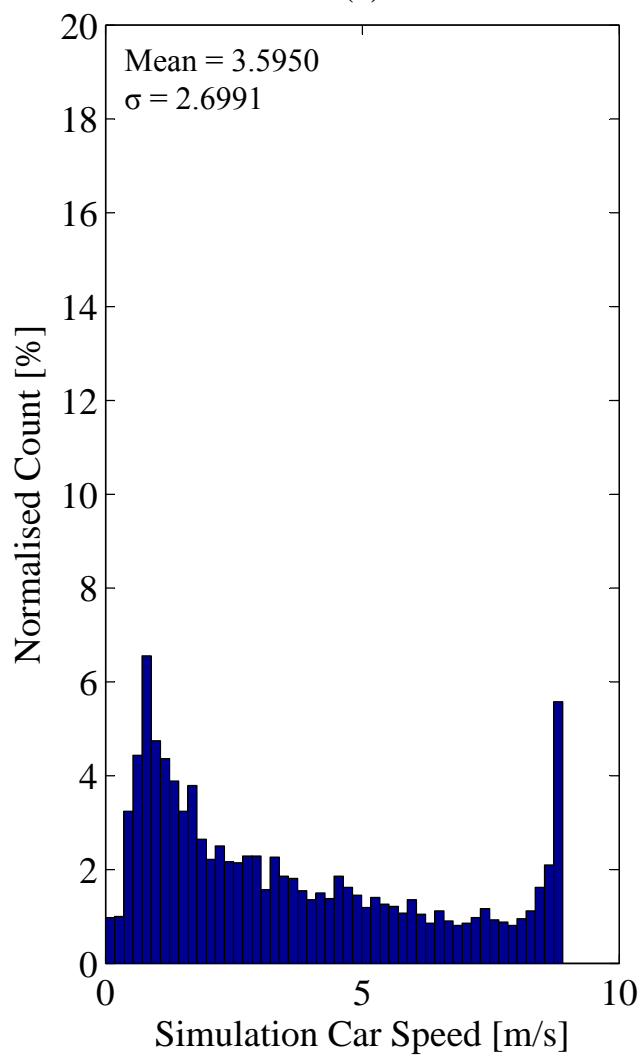

(b)

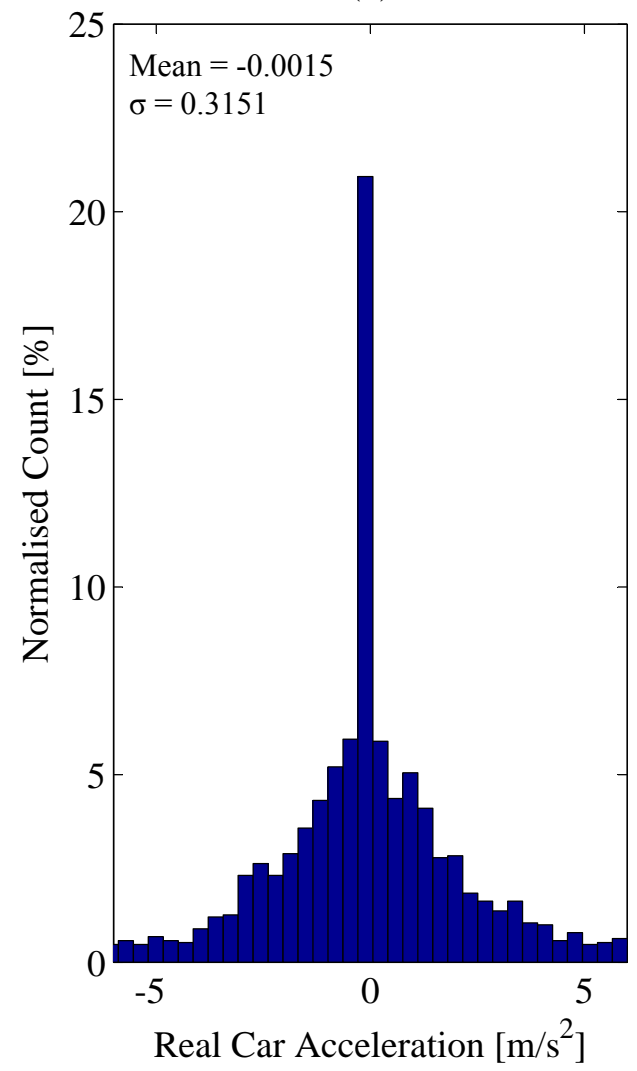

(d)

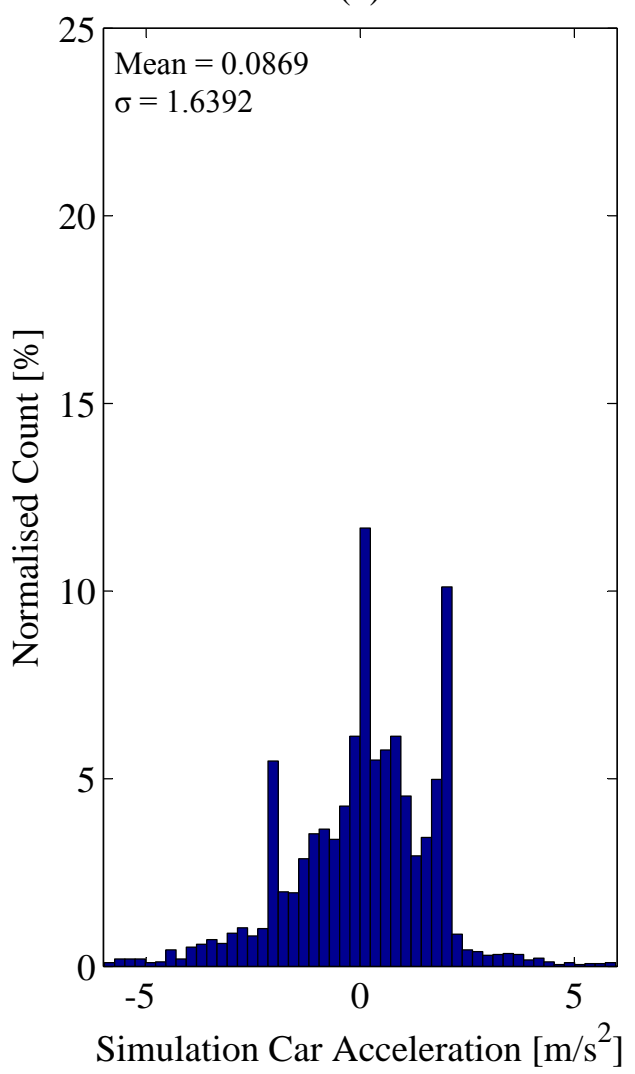

FIGURE 6 : Speed and acceleration histograms of cars on Exhibition Road (London, UK) According to (a)-(b) Empirical data and (c)-(d) Simulation results 
(a)

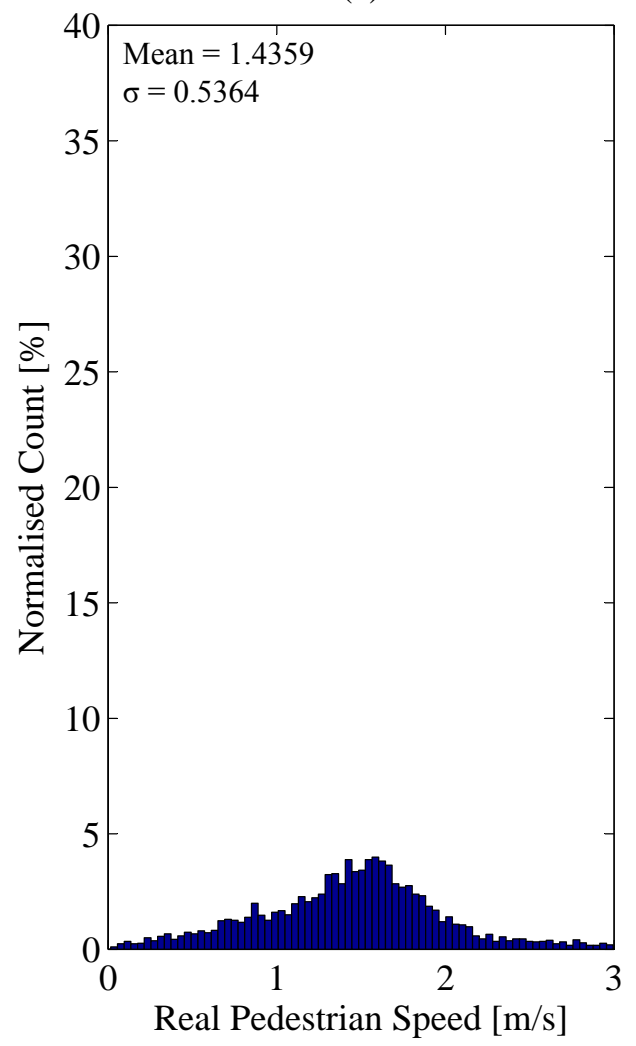

(c)

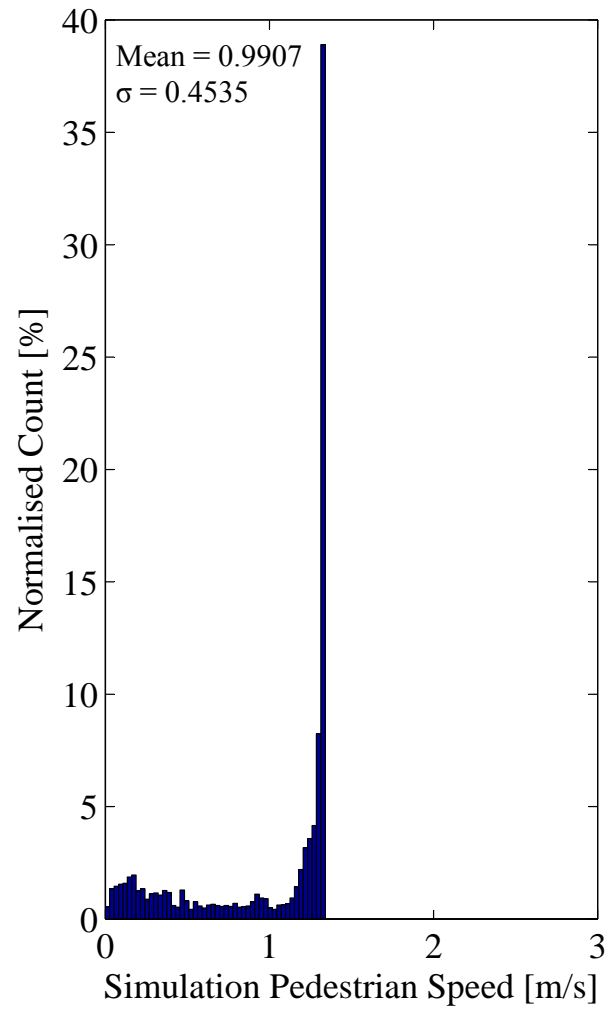

(b)

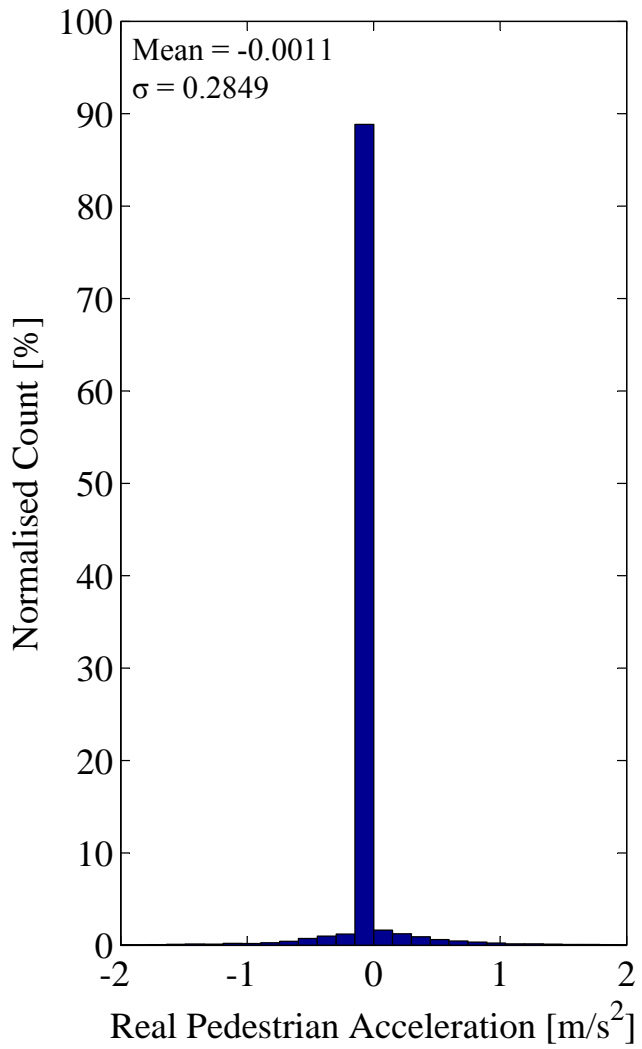

(d)

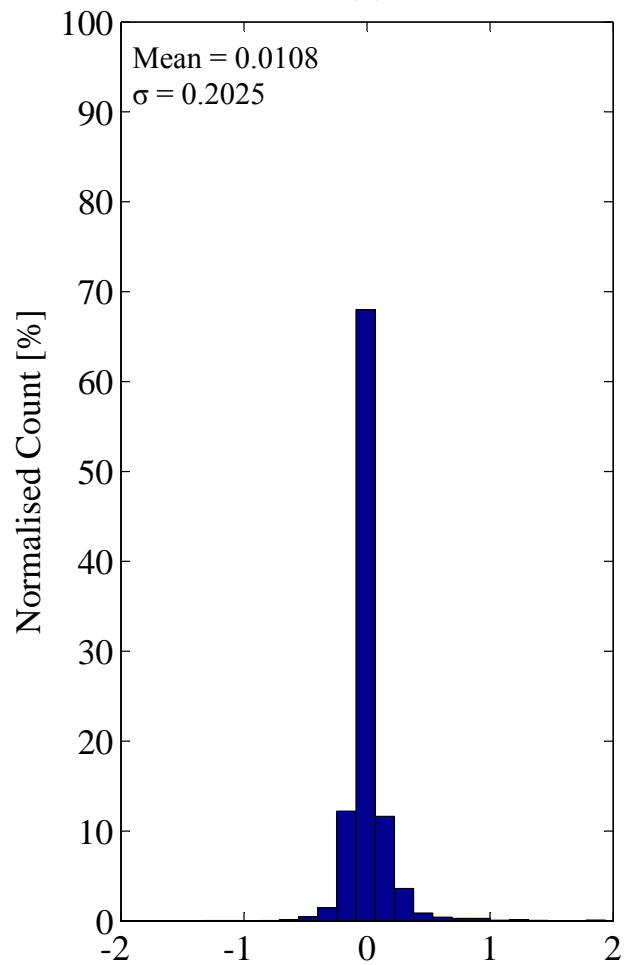

Simulation Pedestrian Acceleration $\left[\mathrm{m} / \mathrm{s}^{2}\right]$

FIGURE 7 : Speed and acceleration histograms of pedestrians on Exhibition Road (London, UK) According to (a)-(b) Empirical data and (c)-(d) Simulation results 
the observed data. Comparing the speed and acceleration distribution of real data and simulation, the patterns are very similar.

It can be concluded that shared space schemes are context-dependent and factors such as the infrastructural design of the environment and the flow and speed of pedestrians and vehicles affect the willingness to share space.

\section{References}

[1] B. Hamilton-Baillie, "Towards Shared Space," Urban Design International, vol. 13, no. 2, pp. 130-138, 2008.

[2] P. Jones and N. Boujenko, "Street Planning and Design Using Link and Place," JOURNEYS Sharing urban transport solutions, vol. 6, pp. 7-14, May 2011.

[3] R. Schonauer, M. Stubenschrott, W. Huang, C. Rudloff, and M. Fellendorf, "Modeling Concepts for Mixed Traffic: Steps towards a Microscopic Simulation Tool for Shared Space Zones," Research Record: Journal of the Transportation Research Board, vol. 2316, no. 1, pp. 114-121, 2012.

[4] M. Shahin, "Pedestrian Behaviour with Mixed Traffic in Developing Countries," Traffic Engineering and Control, vol. 47, no. 8, pp. 303-309, 2006.

[5] G. Tiwari, S. Bangdiwala, A. Saraswat, and S. Gaurav, "Survival Analysis: Pedestrian Risk Exposure at Signalised Intersections," Transport Research Part F, vol. 10, no. 2, pp. 77-89, 2007.

[6] R. Rastogi, S. Chandra, J. Vamsheedhar, and V. Das, "Parametric Study of Pedestrian Speeds at Midblock Crossings," Journal of Urban Planning and Development, vol. 137, pp. 381-389, 2011.

[7] B. Kadali and P. Vedagiri, "Modelling Pedestrian Road Crossing Behaviour under Mixed Traffic Condition," European Transport, vol. 55, pp. 1-17, 2013.

[8] B. Li, “A Model of Pedestrians' Intended Waiting Times for Street Crossings at Signalized Intersections," Transportation Research Part B: Methodological, vol. 51, pp. 17-28, 2013.

[9] D. Helbing, R. Jiang, and M. Treiber, "Analytical Investigation of Oscillations in Intersecting Flows of Pedestrian and Vehicle Traffic," Physical Review E, vol. 72, no. 4, pp. 046130104613010, 2005.

[10] C. Pretto, H. Cybis, and A. Jacobsen, "A Multi-Layer Simulation Model for Vehicle and Pedestrian Interaction," in TRB Annual Meeting, 2011.

[11] D. Sun, S. Ukkusuri, R. Benekohal, and S. Waller, "Modeling of Motorist-Pedestrian Interaction at Uncontrolled Mid-block Crosswalks,” Urbana, vol. 51, p. 61801, 2002.

[12] J. Sun, Y. Yang, and H. Wang, "Development and Application of a Simulation-Enhanced Platform for Pedestrian Crossing Behaviors Experiment," in Transport Research Board, 2011. 
[13] T. Wang, J. Wu, P. Zhang, and M. Mc, "Study of Pedestrians' Gap Acceptance Behavior when They Jaywalk outside Crossing Facilities," in International IEEE Annual Conference on Intelligent Transportation Systems, 2010.

[14] X. Zhang and G. Chang, "A Pedestrian-Vehicle Mixed Flow Simulation Model for Urban Congested Intersections," in Transportation Research Board, 2012.

[15] M. Ottomanelli, G. Iannucci, and D. Sassanelli, "Simplified Model for Pedestrian-Vehicle Interactions at Road Crossings Based on Discrete Events System," Transportation Research Record: Journal of the Transportation Research Board, vol. 2316, no. 1, pp. 58-68, 2012.

[16] M. Ishaque and R. Noland, "Trade-offs between Vehicular and Pedestrian Traffic using Micro-Simulation Methods," Transport Policy, vol. 14, pp. 124-138, 2007.

[17] C. Boenisch and T. Kretz, "Simulation of Pedestrians Crossing a Street," in Traffic and Granular Flow'09, 2009.

[18] B. Anvari, M. Bell, A. Sivakumar, and W. Ochieng, "Modelling shared space users via rulebased social force model," Transportation Research Part C: Emerging Technologies, vol. 51, pp. 83-103, 2015.

[19] The Royal Borough of Kensington and Chelsea, "Exhibition Road," January 2011. Available at http://www.rbkc.gov.uk/subsites/exhibitionroad.aspx.

[20] B. Anvari, "A Mathematical Model for Driver and Pedestrian Interaction in Shared Space Environments," in Universities’ Transport Study Group, 2012.

[21] B. Anvari, W. Daamen, V. Knoop, S. Hoogendoorn, and M. Bell, "Shared Space Simulation Based on Social Forces and Distance Potential Field," in International Conference on Pedestrian and Evacuation Dynamics, 2012.

[22] B. Anvari, M. Bell, P. Angeloudis, and W. Ochieng, "Prediction of Potential Conflicts and Velocity Optimisation of Long-range Collision Avoidance for Force-based Shared Space Simulation," in International Conference on Pedestrian and Evacuation Dynamics, 2014.

[23] T. Kretz, C. Bonisch, and P. Vortisch, "Comparison of Various Methods for the Calculation of the Distance Potential Field," Pedestrian and Evacuation Dynamics, no. 5, 2008.

[24] D. Helbing, I. Farkas, and T. Vicsek, "Simulating Dynamical Features of Escape Panic," Nature, vol. 407, pp. 487-490, 2000.

[25] K. Lewin, Field Theory in Social Science: Selected Theoretical Papers. New York: Harper \& Row, 1951.

[26] T. Lee, An Agent-Based Model to Simulate Motorcycle Behaviour in Mixed Traffic Flow. PhD thesis, Centre for Transport Studies, Department of Civil and Environmental Engineering, Imperial College London, 2007.

[27] M. Apel, "Simulation of Pedestrian Flows Based on the Social Force Model Using the Verlet Link Cell Algorithm,” Master's thesis, Poznan University of Technology, 2004. 
[28] B. Kerner, Introduction to Modern Traffic Flow Theory and Control:The Long Road to ThreePhase Traffic Theory. Berlin: Springer, 2009.

[29] A. Johansson, D. Helbing, and P. Shukla, "Specification of a Microscopic Pedestrian Model by Evolutionary Adjustment to Video Tracking Data," Physics and Society, vol. 9, no. 10, pp. 271-288, 2008.

[30] M. Campanella, S. Hoogendoorn, and W. Daamen, "Quantitative and Qualitative Validation for General Use of Pedestrian Models," in Pedestrian and Evacuation Dynamics, 2012.

[31] D. Parisi and C. Dorso, "Morphological and Dynamical aspects of the Room Evacuation Process," Physica A: Statistical Mechanics and its Applications, vol. 385, no. 1, pp. 343355, 2007.

[32] I. Kaparias, M. Bell, A. Miri, C. Chan, and B. Mount, "Analysing the Perceptions of Pedestrians and Drivers to Shared Space," Transportation Research Part F: Traffic Psychology and Behaviour, vol. 15, no. 3, pp. 297-310, 2012. 